\title{
The outcomes agenda: contribution of the UK clearing house on health outcomes
}

\author{
A F Long, P Dixon, R Hall, R A Carr-Hill, T A Sheldon
}

The UK Clearing House for Information on the Assessment of Health Services Outcomes has only recently started an advisory service, but it is already apparent from the inquiries received that considerable confusion seems to exist about what is and is not health outcome measurement. Indeed, staff at the clearing house are frequently asked to provide basic definitions and to answer fundamental questions, such as what health outcomes can and should be measured. These are complex issues and there are often no "correct" answers.

Any definitional efforts run the risk of introducing further terms and distinctions into an already confused topic. The task would be easier if the slate could be wiped clean and words such as process, input, output, and outcome used afresh, but short of introducing an entirely new terminology it is hard to avoid words which are already in common and highly ambiguous usage. There is little alternative to re-using them, albeit cautiously. There are also limits to the value of any formal definition. It is one thing to understand the theoretical and methodological requirements for "true" outcome measurement, quite another to apply them in real life where effects due to different care processes cannot be separated or where it may be impracticable to control for severity or comorbidity. Despite the many practical obstacles to applying a rigorous measurement model such a model is important if there are to be methodological standards to guide data collection and assessment of health outcomes, both in research and in routine practice. This article tries to indicate our thinking on these matters. It makes no claim to be definitive; like the topic, it is continually developing.

\section{What are health outcomes?}

There are several parts to the definition of health outcomes. Outcomes, in general, are the results (effects) of processes: they are that part of the output of a process which can be attributed to the process. The issue of attribution is too often overlooked, and the measurer assumes that a process is responsible for all its outputs. Health outcomes are the effects on health of any type of process. NHS and private health care services will obviously have health outcomes, but so will other areas such as housing, social services, and employ- ment. Health service outcomes are the effects of health services. Mostly these will have effects on health, but they also include patients' satisfaction with, and attitude to, the services. They may refer to the outcome for an individual or for the local community or a wider population.

Health outcome measurement is thus concerned with the health outcomes of all types of services. Because of its sponsorship, the clearing house spends most of its time on health outcomes due to health care services, either in the form of outcomes for individual patients or for populations and thus wider health gains.

\section{What should be measured?}

Traditionally, health outcome measurement concentrated on mortality and related issues such as rates of avoidable deaths. There is now an increasing concern with morbidity and health related quality of life. Instruments are available to measure many of the aspects of health covered by the World Health Organisation's wide ranging definition of the term - that is, a complete state of physical, mental, and social wellbeing. At least four separate aspects of "health" and associated outcome measures may be the centre of attention: quantity of life, health related quality of life, satisfaction with health care, and process based outcome measures (table). In practice, what actually gets measured will largely depend on who wants the data and for what purpose. The critical factor is who sets the measurement agenda - clinicians, managers, researchers, patients, or some combination of these, or other groups.

A further set of questions concerns the choice of health care services for outcome measurement. In theory there are no limits to the types of services that can have their outcomes measured, though, once again, what actually gets measured will reflect the influence of different interests on the measurement agenda. There are, however, serious practical difficulties in evaluating some types of services. These include the problems of measuring outcomes when the service has low level effects (consultations without interventions); when the start and end of treatment are unclear (outcomes for long courses of rehabilitation or continuous Leeds LS2 9PL

Accepted for publication 8 January 1993 
Aspects of health and associated outcome measures

\begin{tabular}{|c|c|c|c|c|c|}
\hline \multirow[t]{2}{*}{ Quantity of life } & \multirow[t]{2}{*}{$\begin{array}{c}\text { Process based } \\
\text { outcome measures }\end{array}$} & \multicolumn{3}{|c|}{$\begin{array}{c}\text { Quality of life } \\
\text { Health related quality of life } \\
\text { (Dimensions of health) }\end{array}$} & \multirow[t]{2}{*}{ Satisfaction with health care } \\
\hline & & Physical & Social & Mental & \\
\hline Mortality & Readmission rates & Measures of impairment & Measures of handicap & Measures of depression & Patient satisfaction surveys \\
\hline $\begin{array}{l}\text { Avoidable premature } \\
\text { mortality }\end{array}$ & $\begin{array}{l}\text { Relapses } \\
\text { Complications }\end{array}$ & $\begin{array}{l}\text { Disease specific measures } \\
\text { Pain scales } \\
\text { Measures of functional status }\end{array}$ & $\begin{array}{l}\text { Measures of social support } \\
\text { Measures of disability }\end{array}$ & $\begin{array}{l}\text { Measures of social } \\
\text { adjustment }\end{array}$ & \\
\hline
\end{tabular}

amelioration of symptoms in chronic conditions); and when there is comorbidity or several treatments are being conducted simultaneously.

There are three important general considerations whatever type of health care process is being evaluated. Firstly, a treatment episode must be clearly defined. Secondly, methods must exist to control for variations in patient characteristics (severity, age, comorbidity), service factors such as casemix, and ways of isolating the effects due to the service to be monitored from those caused by other factors from within and outside the health services. Thirdly, the period of data collection must be long enough to ensure that all relevant effects will appear or that a sufficiently large sample can be studied.

\section{Which instrument and what type of measure?}

A tentative indication of which measures are most commonly being used can be gained from a computerised outcome projects database which has been created by the clearing house following a "call for information" in August 1992 to a range of possible users for details on outcomes projects planned, recently completed, or underway. So far over 400 responses have been received. Over half of the reported studies seem to be using locally developed measures. This is not an encouraging result if a greater standardisation of measurement is envisaged. Of the established measures, the most widely used are the relatively recent multidimensional health profiles, such as the Nottingham Health Profile (NHP) and SF-36 profile (fig 1). However, representatives of a much older set of measures of functional status are still being extensively used; figure 2 lists the ten most frequently used measures. That the EUROQOL index appears on this list may be an indication of current interest in trying to develop measures of health for use in resource allocation.

It is also evident from the projects database that approaches to outcome measurement are very varied. Five types of activity are most frequently reported: assessing a particular intervention in controlled trials, assessing outcomes in the context of routine clinical practice, routine data collection as part of service monitoring, measurement by clinicians for individual patient diagnosis and prognosis, and studies to develop and test properties of outcome measures. Less frequently reported are the use of outcomes measurement in

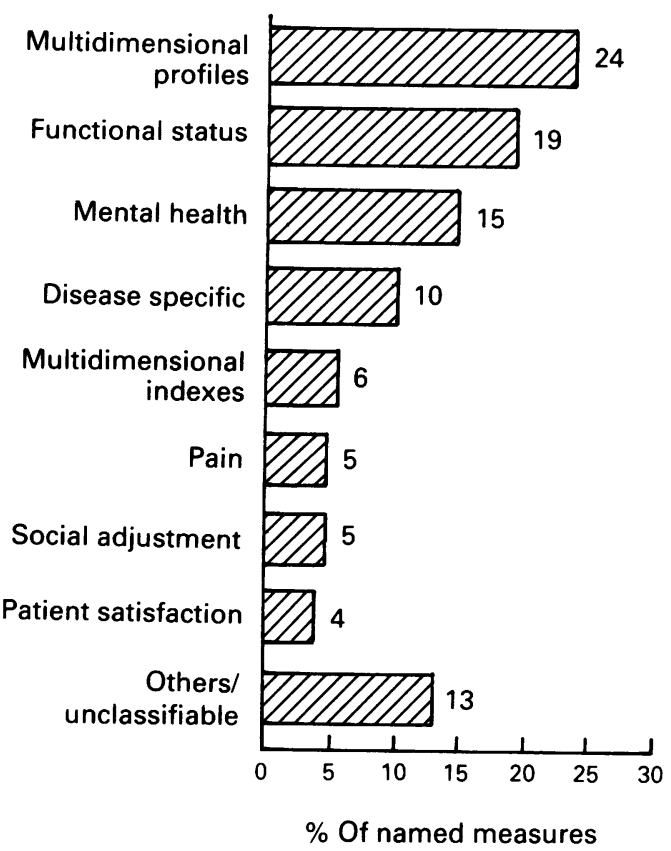

Fig 1 Most widely used measures

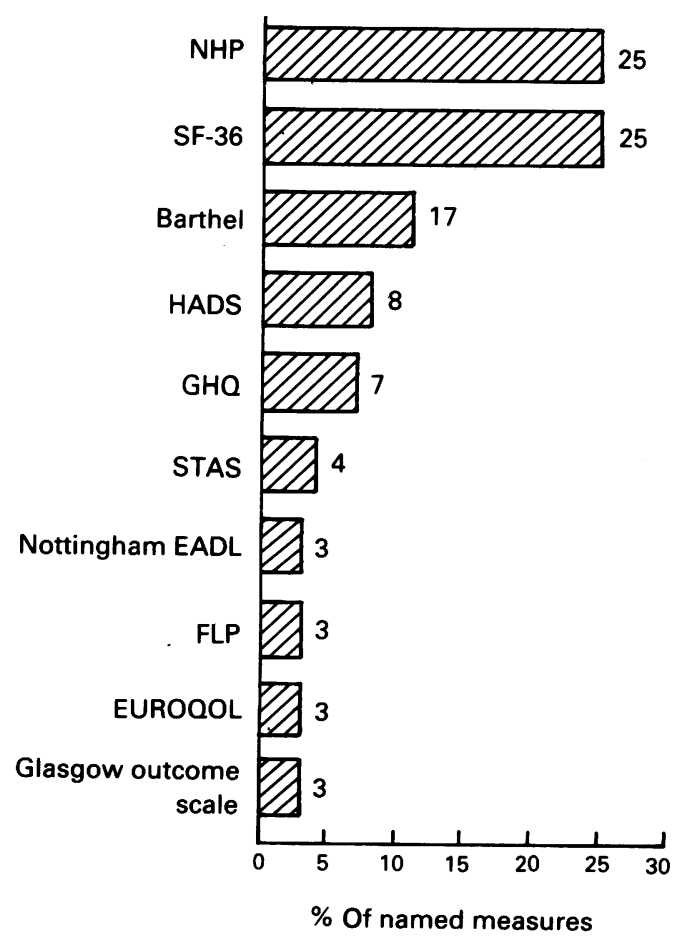

Fig 2 Ten most commonly used measures (NHP=Nottingham health profile, Barthel=Barthel activities of daily living index, HADS=hospital anxiety and depression scale, $G H Q=$ general health questionnaire, $S T A S=$ support team assessment scale, Nottingham $E A D L=i n d e x$ of extended activities of daily living, $F L P=$ functional limitations profile, $E U R O Q O L=$ European quality of life index 
routine service management and in policy making, areas for which, arguably, less measures are available.

The detail provided by the chosen instrument needs to match the intended use. An instrument which provides sufficient detail to make diagnoses and help plan individual care may be entirely inappropriate for informing managerial decision making. Many of the instruments developed for clinical outcome measurement provide results at only an ordinal level of measurement, which cannot be aggregated across groups of patients. The cost of administration is another potentially limiting factor. A long and detailed instrument may be essential in a research setting but far too expensive for routine use.

There are no perfect measures, but it is important that instruments at least conform to some basic methodological criteria. There are three standard requirements. Will the instrument produce the same results if reapplied to the same situation? If it does, it is said to be reliable. Is the instrument really measuring what it purports to? If it is, it is said to be valid. Is the instrument capable of identifying small but clinically significant changes? If so, it is said to be responsive to change. Of the three, responsiveness is the most often overlooked, although it is probably the most important. An instrument which is unable to detect relevant changes is useless for outcome measurement, though it may be excellent for other purposes such as screening or needs assessment. A final consideration when choosing a measure is to ensure that some meaning can be attached to its scores or ratings. The best designed data collection, with well tested instruments, may be of little use if the results cannot be compared with standard values.

\section{An endnote}

Health outcome measurement is fraught with methodological pitfalls, many of which need to be anticipated in planning the data collection. The enormous variety of outcome measurement requires an equally wide range of measurement strategies and designs, based on accepted methodological standards. In any field undergoing rapid expansion fragmentation and duplication of effort are bound to occur. Outcome measurement is no exception. The UK clearing house intends to minimise these problems by encouraging both networking and sound methodological practice.

On the basis of inquiries received and a developing sense of the UK outcomes scene, some general observations are appropriate. Firstly, adopting an outcomes perspective is not just a question of choosing an appropriate measure or set of measures. It involves a long term commitment to evaluation and audit. Secondly, measuring outcomes is not just a matter of data collection. It requires careful consideration of why outcomes are to be assessed, an equally careful search for appropriate measures to do so, and choice of a suitable study design such that the outcome can be attributed to the intervention. Thirdly, developing new measures can be difficult and is frequently costly. Extensive work must be done to establish reliability, validity, and responsiveness. It is thus important to resist the temptation to develop an additional measure or to use items from established measures out of their original context. Before starting to develop a new measure it is essential to see what has already been used in a similar setting or what measures might be borrowed from related areas.

The clearing house has a major role in developing methodological standards in the outcomes area. To this end, work has already started on developing a guide to the routine use of outcome measures to help purchasers, providers, and novice researchers and on devising workshops to raise awareness about outcomes and outcome assessment. Future activities will include systematic reviews of commonly used generic health status profiles including the NHP, SF-36, and functional limitations profile and outcome measures within specific conditions such as rheumatoid arthritis and stroke. These activities form part of a dissemination effort which includes a quarterly newsletter to inform users of the work and activity of the clearing house and of work in the outcomes area in the NHS more broadly.

Finally, the clearing house is actively encouraging networking between those involved in outcome measurement as part of routine practice and within a research setting. By maintaining an up to date database of outcome measurement activities it is possible to put inquirers in touch with others working in the same field or with those using similar measures, to avoid duplication and to share experiences. For this networking to succeed the clearing house must be kept informed of ongoing and planned activities in the outcomes area (a proforma for supplying project details is included below). This should facilitate the longer term aim of developing an outcomes culture within the health services.

The first issue of the newsletter Outcomes Briefing has been published. It includes information on the activities of the clearing house; issues and instruments in outcomes measurement; invited contributions; and a round up of news from conferences, user groups, and the UK health departments. A copy will be sent to directors of quality assurance, directors of public health, research and development managers, medical audit coordinators, general managers of family health services authorities, medical audit advisory groups and in addition, to all those who sent details of outcomes work to the clearing house in early March. A limited number of extra copies are available; details from Claire Prest at the clearing house (tel 0532459034 ext 2179). 
- It is essential that all those involved in outcomes related work, in clinical and medical audit, and in health services research more generally, register with the clearing house. Those involved in particular outcome projects are encouraged to provide regularly updated details and planned developments to the clearing house by completing the proforma below.

UK Clearing House for Information on the Assessment of Health Services Outcomes: project/activity data proforma

Project title:

Brief description:

Aims/objectives of project:

Study group (for example, children, elderly, diabetic patients):

Setting (inpatient, outpatient, community, general practice):

Outcome measures used:

Project duration:

Start date:

Funding source:

Contact name:

Address:

Tel:

The clearing house information team may contact you for further information.

Please return with any project publications/reports to:

Robert Hall,

Information Manager,

UK Clearing House for Information on the Assessment of Health Outcomes,

Nuffield Institute for Health Services Studies,

71-75 Clarendon Road,

Leeds LS2 9PL

Tel 0532459034 ext 2179; fax 0532460899 